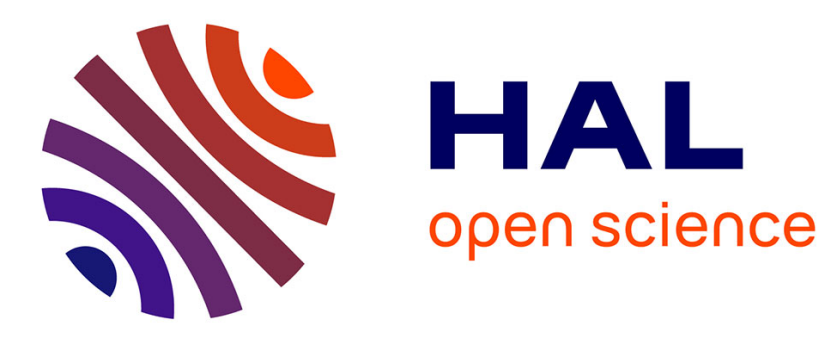

\title{
Ethnologie, racisme et médias
}

Catherine Choron-Baix, Sylvie Fainzang, Jacques Gutwirth, Liliane

Kuczynski, Anne Raulin, Maurice Duval

\section{To cite this version:}

Catherine Choron-Baix, Sylvie Fainzang, Jacques Gutwirth, Liliane Kuczynski, Anne Raulin, et al.. Ethnologie, racisme et médias. Journal des anthropologues, 1990, 42 ("Quelles formations à l'anthropologie? ", coordonné par Marc-Eric Gruénais et Catherine Quimin, pp.125-130. halshs00010536v2

\section{HAL Id: halshs-00010536 \\ https://shs.hal.science/halshs-00010536v2}

Submitted on 10 May 2008

HAL is a multi-disciplinary open access archive for the deposit and dissemination of scientific research documents, whether they are published or not. The documents may come from teaching and research institutions in France or abroad, or from public or private research centers.
L'archive ouverte pluridisciplinaire HAL, est destinée au dépôt et à la diffusion de documents scientifiques de niveau recherche, publiés ou non, émanant des établissements d'enseignement et de recherche français ou étrangers, des laboratoires publics ou privés. 


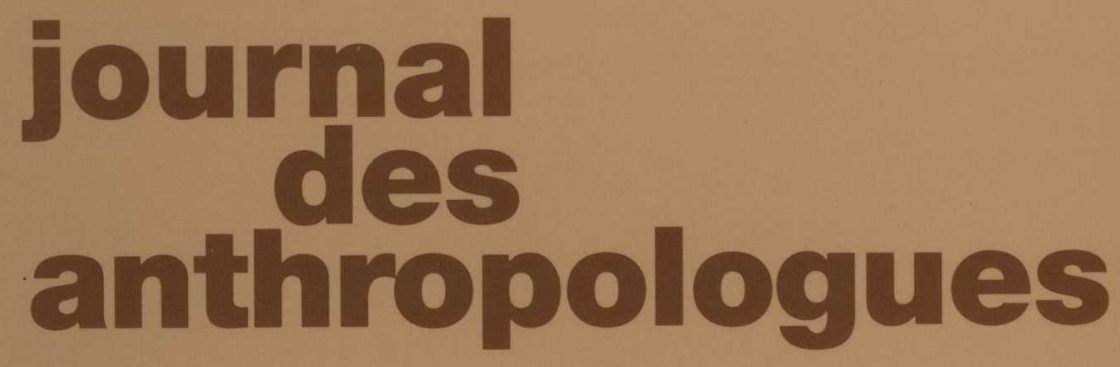

\section{2 \\ déc. 1990}

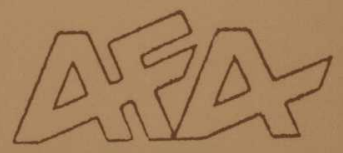




\title{
ETHNOLOGIE, RACISME ET MEDIAS
}

\author{
Catherine BAIX, Sylvie FAINZANG, Jacques GUTWIRTH, \\ Liliane KUCZYNSKI, Anne RAULIN et Maurice DUVAL
}

En tant qu'ethnologues (ou anthropologues, comme on le dit aussi), nous sommes confrontés scientifiquement à la diversité ethnique, religieuse, culturelle, etc. Or à ce sujet, les médias usent souvent de mots, de concepts tels ceux de "communauté" et d' "'identité", dont aucune réflexion critique ne tempère l'utilisation schématique et simplificatrice. Ce fait trouve une résonance particulière actuellement en France, où racisme et xénophobie misent précisément sur l'incompréhension, les préjugés, les malentendus. C'est pourquoi nous considérons qu'il est de notre devoir d'intervenir dans ce débat, auprès de différents publics, et de contribuer à éviter que ne se perpétuent amalgames et confusion.

\section{L'UTILISATION DU TERME "COMMUNAUTE"}

Ce concept accordéon s'il en est, puisqu'il peut tout aussi bien désigner la CEE qu'un petit groupe aussi restreint et sélectif qu'une communauté monastique, semble à première vue présenter les avantages d'une réelle élasticité. Mais à y regarder de plus près, l'usage abusif de ce terme par la presse et les médias, et même dans la recherche, peut mettre mal à l'aise. L'expression de certains individus, de certaines associations est ainsi établie comme la "voix de la communauté" - juive ou musulmane, asiatique ou maghrébine. Comme si, derrière certaines appartenances religieuses ou "ethniques" se constituait nécessairement toute une vie communautaire dont l'homogénéité se révélerait à certaines occasions par un porte-parole, par "une seule voix". A cette vision unanimiste - tous les membres de la communauté rangés comme un seul homme derrière leur 
représentant - l'ethnologue restitue une prolifération de positions faites de divergences profondes ou de nuances infinies, le plus souvent irréductibles aux yeux des intéressés. Il effectue ainsi une "ethnologie des opinions" par laquelle le "comment" de l'appartenance communautaire se trouve posé. Car, dans les situations complexes qui prévalent de nos jours, le rattachement à une communauté est beaucoup plus la manifestation d'une volonté, privilégiant certains liens sociaux au détriment d'autres affinités, que l'héritage d'une tradition en droite ligne. Autant dire que cette question de l'appartenance communautaire revient à l'individu qui la traite comme il peut, en jouant de ses particularités - lesquelles incluent son aspect physique, son sexe, sa position dans la fratrie (aîné ou cadet), ses acquis intellectuels, ses origines sociales (citadines ou rurales), son itinéraire professionnel passé ou à venir, et ce de façon fort peu mécanique; d'autant moins que les conjonctures politiques, économiques ou sociales dessinent périodiquement le sens de ces appartenances en faisant évoluer la nature des relations entre ces différents paramètres communautaires.

\section{LES MULTIPLES IDENTITES}

Pas plus que de la notion de communauté, on ne peut avoir de l'identité une conception fixiste. L'ethnologie et l'histoire nous apprennent qu'il existe plusieurs niveaux d'identité: celle qui n'est que façade (exemple: les Marranes), celle que l'on s'approprie provisoirement et dont on change, tout comme de nom (exemple de certaines cultures d'Afrique de l'ouest, où les acteurs sociaux manipulent patronyme et ethnonyme au gré de stratégies politiques diverses), celle qui est refoulée car considérée comme non valorisante, celle que peut engendrer l'histoire avec ses migrations forcées, ses changements de nationalité imposés, etc.

L'identité peut aussi être vécue positivement ou négativement (exemple: se présenter comme "zonard" peut exprimer la réalité d'une exclusion). Elle peut encore se déterminer selon des références très diverses, l'appui sur une "ethnie" ou une culture n'étant en rien le seul possible.

Que cela relève de l'explosion de l'individualisme ou, plus en amont, $\mathrm{du}$ fait que les liens soudant les sociétés contemporaines sont moins 
contraignants, plus abstraits que dans les sociétés dites traditionnelles, on peut constater que chacun circule entre des réseaux sociaux ou des fragments de réseaux (politiques, familiaux, culturels, syndicaux, professionnels, etc.) parmi lesquels il se constitue autant d'identités partielles ou croisées.

Mais à cette lumière, il ne serait pas vain de réexaminer les sociétés "traditionnelles": on découvrirait certainement qu'elles induisent une identité bien moins univoque qu'on ne l'a souvent prétendu. A cela, au moins une raison décisive, de nature historique: toute culture manifeste, dans sa constitution et son évolution, une grande créativité, où président bricolages, synthèses et cumuls. Il y a là autant d'ouvertures à de nouvelles compositions, tant sociales qu'individuelles.

Il est, alors, d'autant plus paradoxal de continuer à projeter sur autrui une identité monolithique et cette attribution fantasmatique vécue tel un "trop d'identité" (exemple: "les Maghrébins", "les Antillais", "les Juifs") est bien souvent l'expression du racisme.

Ce bloc dans lequel on enferme ainsi les "autres" a pour corollaire d'enfermer ceux qui profèrent ces généralisations dans le même "bunker" d'une identité fallacieuse ("nous les Français") : c'est faire preuve d'une amnésie historique et géographique regrettable, et d'une négation de ce qu'il y a en chacun de composition. A cet égard, une réflexion non seulement sur "les autres" mais aussi sur soi paraît indispensable. Les études d'anthropologie urbaine confirment que nous pratiquons, les uns et les autres, déplacements d'identités, recherches et certitudes provisoires. Nous sommes tous faits de déchirures, de ruptures, d'ancrages multiples. L'Identité" est une construction tout aussi théorique que le Premier Homme ou la Première Langue.

\section{L'ENSEIGNEMENT DE L'ETHNOLOGIE}

L'ethnologie présente un atout majeur dans le débat actuel et pour la dénonciation des falsifications de toutes sortes : pratiquant la description minutieuse de l'organisation matérielle et symbolique des sociétés humaines, elle en fait apparaître toute la complexité. Elle est, en cela, à l'opposé de maints discours ambiants, fondés sur des raccourcis et des approximations, sujets à toutes les manipulations. Peut-être ce souci du 
détail juste et de présentation scrupuleuse des faits culturels freine-t-illa diffusion des travaux ethnologiques? Il est certes plus facile de retenir d'une culture ses aspects les plus spectaculaires, fussent-ils présentés hors contexte, plutôt que d'en restituer la profondeur historique ou les systèmes de significations. Pourtant c'est précisément cet attachement à la présentation approfondie d'un dossier, à l'analyse et à la compréhension de la logique des situations examinées, qui peut barrer la route aux contrevérités.

L'ethnologie doit dès lors faire connaître sa démarche et ses résultats auprès d'un large public. Son enseignement, à différents niveaux, en est un moyen majeur. Il peut prendre des formes adaptées à chacun des auditoires visés. En particulier, dès le second degré, il doit être dispensé à l'ensemble de la population scolaire, comme un élément indispensable dans l'éducation des futurs citoyens. Dans le contexte social et culturel actuel, un minimum d'entraînement au regard ethnologique permet une définition de soi et des autres plus sereine. L'école peut utiliser des textes ethnologiques de qualité, des documents audiovisuels, les ressources des musées et des expositions ethnographiques pour y préparer. Ces supports pédagogiques offrent un grand intérêt éducatif, à condition qu'ils soient mis à profit par des formateurs compétents. On peut aussi tirer le meilleur parti de la pluralité des cultures représentées dans les établissements scolaires, en suscitant chez les élèves un intérêt, une ouverture à des façons d'être autre, à des modes de pensées qui ne menacent nullement "notre" identité, en vérité déjà si composite.

Le champ d'exploration de l'ethnologie ne se limite pas aux sociétés lointaines et aux cultures "traditionnelles", mais comprend aussi, et de plus en plus, les sociétés occidentales et leurs transformations sociales et culturelles. Une éducation moderne doit prendre en compte toutes ses connaissances sur le passé et le présent, le proche et le lointain. L'ethnologie, parce qu'elle est avant tout recherche d'une connaissance approfondie et précise de l'autre, ne doit plus être absente de l'école. 


\section{CONNAISSANCE DES AUTRES ET REFUS DE L'IDEOLOGIE RACISTE}

La possibilité d'accepter l'étranger passe et par l'acceptation de sa différence et par la reconnaissance de ce qui fait de lui un autre nousmême. Le rôle de l'anthropologie est donc, par-delà la mise en évidence de la spécificité de chaque culture et de chaque société, de dégager l'unité de l'homme. Ce qui nous rassemble doit être mis en relief; se focaliser sur les différences et y enfermer les autres, c'est les faire dépendre exclusivement de valeurs qui nous sont étrangères et en conséquence leur interdire l'accès à notre propre humanité. Or si certains traits nous distinguent, d'autres, beaucoup plus importants, sont communs et c'est précisément ceux-là qui font de nous tous des êtres de droit.

Il convient de souligner à cet égard les dangers que présente une pseudo-ethnologie mystifiante qui ferait de l'étranger l'objet de nos fantasmes - de nos peurs, de nos désirs, de nos fascinations -, alors même que la connaissance de l'étranger est ce qui doit permettre de prendre des distances à l'égard de nos propres pratiques: la connaissance de l'étranger comme condition de la connaissance de soi.

C'est en cela qu'il est nécessaire de "dés-exotiser" les sociétés plus ou moins lointaines, comme il est nécessaire de ne pas voir en l'autre que l'autre.

L'ethnologie n'est certes pas un rempart absolu contre le racisme et ne résout évidemment pas tous les problèmes. De plus certains ethnologues (aujourd'hui très minoritaires) n'hésitent pas à publier des "travaux" qui, se parant du discours de la science, sont en vérité le support de positions racistes. Mais une meilleure connaissance des autres peut sûrement contribuer à juguler la propagation des idées racistes.

Il y aurait, toutefois, quelque paradoxe à présenter des recherches rigoureuses dans des publications professionnelles et, dans le même temps, à laisser se répandre, dans l'opinion publique, sans réagir, ce racisme venu notamment de l'extrême-droite.

La propagation de ce discours falsificateur repose sur la conjugaison d'émetteurs, de diffuseurs et de récepteurs. Peut-on empêcher les émetteurs (l'extrême-droite et ses émules) ? Sans doute (la loi reconnaît 
des délits d'opinion), mais c'est l'affaire des citoyens, des politiques, des juristes avant celle des ethnologues. Peut-on faire obstacle à la réception de ces contre-vérités par ceux auxquels, pour diverses raisons, elles sont bien utiles? L'ethnologue ni ses travaux n'ont pour objet la résolution de difficultés existentielles.

Reste les diffuseurs, les médias, relais de ce discours. Ils montrent parfois une complaisance douteuse à son égard, même si nombre de journalistes ne sont, sur ce plan, guidés que par leur conscience professionnelle. Or, lorsque les médias audio-visuels donnent dans certaines émissions la parole aux tenants du racisme, nous pouvons, en tant que scientifiques, réclamer que celles-ci soient systématiquement accompagnées ou suivies de commentaires et discussions par des historiens, des sociologues, des ethnologues ou autres spécialistes du sujet traité. Ceux-ci auraient ainsi l'opportunité de relever les falsifications auxquelles se livre l'extrême-droite. Ce droit de réponse scientifique est indispensable pour lutter contre la désinformation.

Dans le même esprit, nous suggérons la création auprès des chaînes de télévision, de comités scientifiques composés de spécialistes de différentes disciplines, dont le rôle serait de veiller à rétablir, au détour des programmes ici incriminés, quelques vérités moins simplistes.

Vocabulaire plus juste, enseignement de l'ethnologie et intervention scientifique sur les médias nous paraissent des moyens précieux pour le combat contre le racisme et la xénophobie, poisons de nos sociétés, qui, si on n'y prend garde, risquent de nous conduire au totalitarisme avec toutes ses conséquences funestes. 
oai:halshs.archives-ouvertes.fr:halshs-00010536_v1

Numéro épuisé

Fac-similé déposé en Archives ouvertes avec l'autorisation de la revue

Scan et reconnaissance de caractères : Eliane Daphy (avril 2006)

Source : exemplaire personnel Eliane Daphy

Ce numéro a été coordonné par Marc-Eric Gruénais et Catherine Quiminal.

La fabrication a été assurée par Béatrice Schatz (Société IODE) et la couverture réalisée par Mbaye Diouf.

Nous tenons à remercier les auteurs des manuscrits qui nous sont envoyés et qui sont autant de contributions à la vie de l'Association.

La forme et le contenu des articles n'engagent que la responsabilité de leurs auteurs. 
oai:halshs.archives-ouvertes.fr:halshs-00010536_v1

Numéro épuisé

Fac-similé déposé en Archives ouvertes avec l'autorisation de la revue Scan et reconnaissance de caractères : Eliane Daphy (avril 2006)

Source : exemplaire personnel Eliane Daphy

\title{
COLLECTIF DE REDACTION
}

\author{
Marie-Christine ANEST -COUFFIN \\ Claude ARDITI \\ JeanCOPANS \\ Rita CORDONNIER \\ Nicole ECHARD \\ Marie·Paule FERRY \\ Jeanine FRIBOURG \\ Marc-Eric GRUENAIS \\ Jean HANNOYER \\ Danièle KINTZ \\ Annie LE PALEC \\ Olivier MASCLET \\ Catherine QUIMINAL \\ Monique SELIM \\ Alex-Louise TESSONNEAU
}

Toute correspondance doit être adressée à :

Journal des anthropologues

A.F.A. - E.H.E.S.S.

1, rue du 11 Novembre

92120 MONTROUGE

Tél. : (1) 4092 173d

Permanence le mardi après-midi

Voir présentation de la revue et sommaires des numéros sur le site inttp./Wwww.ala.msh-parts.fitfournal.htmit| 


\section{DOSSIER - QUELLES FORMATIONS A L'ANTHROPOLOGIE ?}

Quelles formations à l'anthropologie? M.-E. GRUENAIS et C. QUIMINAL Entretien avec Emmanuel Terray M.-E. GRUENAIS et C. QUIMINAL Formation doctorale d'ethnologie et de sociologie comparative. Louis MALLART-GUIMERA

Le magistère de Sciences sociales de

Paris V - Sorbonne

Dominique DESJEUX et Sophie TAPONIER "Université et musée : une collaboration nécessaire". Le café des ethnologues.

Rencontres étudiantes Daniel TERROLLE Le CNRS et l'anthropologie appliquée : un point de vue Olivier MASCLET L'anthropologie dans la formation des enseignants Gérard LENCLUD Du technicisme à l'accompagnement de dynamiques sociales : la "capitalisation" du G.R.D.R. Notes à propos de l'enseignement de l'anthropologie dans une organisation non gouvernementale médicale Dominique MARTIN

\section{HOMMAGES A MICHEL LEIRIS}

Encens pour Berhane Michel LEIRIS

De l'ethnologie considérée comme une tauromachie Joseph TUBIANA Soixante ans d'amitié Jacques FAUBLEE

Michel Leiris devant le colonialisme Claude ARDITI Bibliographie sélective de Michel Leiris Marie-Paule FERRY

\section{ANTHROPOLOGIE VISUELLE}

Société Française d'Anthropologie Visuelle....

Burkina Faso, en pays lobi, tournage du film "

"Les mémoires de Binduté Da". Michèle FIELOUX

Regards sur les sociétés européennes. Jacques LOMBARD

Séminaires, conférences, festivals 1990 Daniel PELLIGRA Colette PIAULT

\section{NOUVELLES DE LA PROFESSION}

Que viva la reforma! Bertrand GERARD Offres d'emploi La disparition d'une formation en anthropologie Jean-Pierre CASTELAIN 